\title{
Vendaje neuromuscular como herramienta complementaria a la terapia respiratoria de adultos con asma
}

\author{
Kinesio taping as a complementary tool to respiratory therapy adults with asthma \\ Ivette Giselle Ramos Bendezú ${ }^{1}$, Angelique Katterine Delgado Guzmán ${ }^{1}$, Lizet Roxana Vitón Rubio ${ }^{1}$, \\ Elizabeth Cecilia Meléndez Olivari ${ }^{1, a}$, José Luis Rojas Vilca ${ }^{1, b}$
}

\section{RESUMEN}

Objetivo: Determinar el efecto del vendaje neuromuscular (VNM), como herramienta complementaria a la terapia respiratoria en adultos con asma, sobre parámetros respiratorios fisiológicos (frecuencia cardiaca, frecuencia respiratoria, saturación de oxígeno y patrón respiratorio) y espirométricos (capacidad vital, flujo espiratorio máximo, volumen espiratorio forzado). Material y métodos: Estudio de tipo observacional, comparativo, longitudinal y prospectivo. Se incluyó 40 pacientes asmáticos adultos atendidos en el programa de Rehabilitación Cardiorrespiratorio de un hospital general, quienes recibieron la terapia respiratoria convencional, seleccionando por conveniencia 20 pacientes a quienes se les aplicó adicionalmente el VNM y 20 a quienes no. Resultados: El grupo que recibió VNM tuvo mayor mejoría en los parámetros respiratorios espirométricos capacidad vital (CV), volumen espiratorio forzado al primer segundo (VEF1) y flujo espiratorio forzado (PEF) $(\mathrm{p}<0,01)$, así como mayor incremento en la $\mathrm{SO} 2(\mathrm{p}<0,01)$, en comparación con quienes no se les aplicó. En los parámetros fisiológicos respiratorios frecuencia cardiaca (FC) y frecuencia respiratoria (FR) se encontraron diferencias significativas ( $p<0,01)$. Asimismo, el 100\% de pacientes del grupo que recibió VNM mejoraron su patrón respiratorio de torácico a diafragmático en comparación con el 75\% del grupo que no recibió VNM (prueba exacta de Fisher $=7,059, \mathrm{p}=0.01$ ). Conclusiones: El VNM ha mostrado un efecto beneficioso en la población estudiada como herramienta complementaria a la terapia respiratoria en pacientes asmáticos.

Se recomienda realizar más estudios relacionados a la aplicación de esta técnica en pacientes asmáticos como complemento a la fisioterapia respiratoria, que evalúen la eficacia y seguridad según severidad y grupos etáreos.

PALABRAS CLAVE: Vendaje neuromuscular, asma, rehabilitación respiratoria.

\section{SUMMARY}

Objective: Determine the effect of neuromuscular bandage (VNM) as a complementary tool to respiratory therapy in adults with asthma, on physiological respiratory parameters (heart rate, respiratory rate, oxygen saturation and respiratory pattern) and spirometry (vital capacity, expiratory flow) maximum, forced expiratory volume). Material and methods: Observational, comparative, longitudinal and prospective study. We included 40 adult asthmatic patients treated in the Cardiorespiratory Rehabilitation program of a general hospital, who received conventional respiratory therapy, selecting for convenience 20 patients to whom the VNM was additionally applied and 20 to those who did not. Results: The group that received VNM had greater improvement in spirometric parameters vital capacity (VC), forced expiratory volume to the first second (FEV1) and forced expiratory flow (PEF) ( $p<0.01)$,

Escuela de Tecnología Médica, Facultad de Medicina Alberto Hurtado, Universidad Peruana Cayetano Heredia. Lima, Perú.

a Licenciada en Terapia Física y Rehabilitación; ${ }^{\text {a }}$ Médico-Cirujano 
as well as greater increase in $\mathrm{SO} 2(\mathrm{p}<0.01)$, compared to those who did not. In the respiratory physiological parameters heart rate (HR) and respiratory rate (FR) significant differences were found $(\mathrm{p}<0.01)$. Likewise, $100 \%$ of patients in the group that received VNM improved their respiratory pattern from thoracic to diaphragmatic compared to $75 \%$ of the group that did not receive NOM (Fisher's exact test $=7.059, p=0.01$ ). Conclusions: VNM has shown a beneficial effect in the population studied as a complementary tool to respiratory therapy in asthmatic patients. It is recommended to carry out more studies related to the application of this technique in asthmatic patients as a complement to respiratory physiotherapy, which evaluate the efficacy and safety according to severity and age groups.

KEYWORDS: Kinesiotaping, asthma in adults, respiratory rehabilitation.

\section{INTRODUCCION}

El vendaje neuromuscular es un método que se origina en Asia (Corea y Japón), de la mano del Dr. Kenzo Kase y del Dr. Murai en los años setenta y tras difundirse primero en los ámbitos deportivos de elite internacional, hoy en día su uso es más frecuente y por muchos kinesiólogos o fisioterapeutas de todo el mundo. Entre sus efectos podemos destacar el efecto analgésico que es atribuible a la disminución de la presión intersticial, con lo que se modifica la estimulación o inhibición de diferentes receptores nerviosos y a la liberación de diferentes sustancias (endorfinas, encefalinas) (1). El efecto neuromecánico que actúa a través de la tracción en la piel, incidiendo sobre el tejido celular subcutáneo y las fascias. Este efecto mecánico mínimo pero sostenido en el tiempo, tendría su acción en el tono del músculo sobre el cual se aplica el vendaje (aumentándolo o disminuyéndolo) dependiendo del sentido de la tracción de la piel. El VNM genera un estímulo exteroceptivo que es recogido por los mecanorreceptores ubicados en la piel y las fascias, y es transmitida en sentido aferente hacia el sistema nervioso central que mejora la calidad del movimiento. El estímulo de los propioceptores, que confieren al organismo la capacidad de percibir la posición y el movimiento de sus estructuras sobre todo en el plano músculo esquelético, va a conseguir mandar información permanente sobre el estado artrocinemático de la articulación y así poder influir sobre la postura articular y su estabilidad. Además, tiene efecto sobre la circulación sanguínea y el drenaje linfático, lo cual se debe a la elevación del vendaje sobre la piel y el cambio de presión sobre la linfa haciendo que mejore su drenaje. Es decir, que estos efectos se van a ver reflejados en la reducción de la fatiga muscular, modulando el tono muscular, mejorando la flexibilidad, aumentando la estabilidad articular y la postura, acelerando el proceso de reparación de la lesión, aumentando la circulación sanguínea local y favoreciendo el drenaje de las sustancias de desecho en la zona (2).

Aunque el VNM ha sido más usado en el área deportiva o traumatológica, en los últimos años se ha observado su incursión en otras áreas como la respiratoria, encontrando el uso del VNM como una herramienta positiva. Así lo muestra Couto et al., (3) en su estudio donde evaluaron la acción del VNM en la musculatura respiratoria del diafragma e intercostales externos asociada a la fisioterapia convencional en pacientes del sexo femenino con EPOC. Antes y después de cada sesión de fisioterapia todas las mujeres realizaban las medidas de presión inspiratoria máxima (PImax), presión espiratoria máxima (PEmax), cirtometría (axilar y abdominal), saturación de oxígeno (SpO2) y escala de BORG modificada. Al final del estudio, concluyeron que la mayoría de los pacientes presentó mayor movilidad abdominal y el $60 \%$ de las mujeres reportaron mayor facilidad para realizar las incursiones respiratorias.

Jiménez ${ }_{(4)}$ reportó los resultados de 2 pacientes con hipersecreción bronquial, a quienes colocaron el vendaje neuromuscular en la zona anterior del diafragma durante 5 semanas, en quienes la mecánica respiratoria se modificó y aumentó la movilidad abdominal en lugar del costal superior; se observó mejoría en la movilidad abdominal y la postura, disminuyendo la disnea, el tiraje y periodos de apnea, haciendo el tono muscular más simétrico; se observó que, en días posteriores a la colocación del vendaje la tos era más eficaz, indicativo de que el drenaje mucociliar había aumentado, posiblemente por el incremento de la respiración diafragmática, con lo cual aumentó la eliminación de secreciones, mejoró la sintomatología respiratoria y disminuyó el tiempo dedicado a los ejercicios respiratorios en beneficio de otras técnicas fisioterapéuticas. 
Van Zuilen (5), combinó la terapia inhalatoria con la terapia respiratoria, incluyendo aspiración de secreciones y oxigenoterapia, en los casos más severos, así como diversas maniobras kinésicas. Además, utilizó el vendaje neuromuscular en zona pulmonar, diafragma, músculos escalenos y pectoral mayor, obteniendo una mejoría visible de la forma y movilidad del tórax, el funcionamiento del diafragma, mejoría en la fuerza para toser y secreción del esputo, y disminución de la hipertonía de los músculos escalenos.

Por otro lado, se sabe que el asma es una inflamación crónica de la vía aérea (bronquios), proceso que se asocia a una hiperrespuesta bronquial (6), y donde los músculos de los pacientes asmáticos sufren adaptaciones, entre las cuales se incluyen hipertrofia de los accesorios y de los intercostales y la contracción de los músculos inspiratorios durante la espiración. Estas adaptaciones pueden ocurrir como resultado de los numerosos esfuerzos violentos a los que los pacientes están expuestos, y que son necesarios para que estos músculos aumenten la carga resistiva adicional, representada por la contracción de las vías aéreas, y a la carga elástica, debida al aumento del volumen pulmonar. También se ha evidenciado que la disminución de la fuerza de un músculo en función de su longitud está prevista de acuerdo con las relaciones fuerza-longitud. En el caso de los músculos respiratorios, el aumento del volumen pulmonar puede volver más plano el diafragma, acortando sus fibras, y manteniéndolos en una posición de desventaja mecánica, con la consecuente disminución de la capacidad para desarrollar presión que se manifiesta con sibilancias, disnea, opresión torácica y tos, particularmente por la noche o de madrugada (7). El tratamiento de la enfermedad incluye el uso de corticoides y beta 2 agonistas inhalados (8), y medidas como la fisioterapia pulmonar para mejorar de la capacidad funcional respiratoria, mediante la ejecución adecuada de ejercicios dirigidos a actuar sobre la movilidad de los músculos respiratorios, utilizando ejercicios respiratorios, maniobras posturales y de drenaje. Los programas de rehabilitación respiratoria incluyen maniobras fisioterapéuticas activas en ejercicios respiratorios puros, respiratorios rítmicos con determinado movimiento y movimientos musculares no rítmicos con la respiración; además, procuran la adopción de posturas correctas, aplican técnicas de relajación muscular (en especial del cuello y de la cintura escapular) para prevenir que factores emocionales como la ansiedad pueda aumentar el broncoespasmo, realizan ejercicios respiratorios a partir de las posiciones correctas, rehabilitación de la movilidad costal inferior y diafragmática, disminución de la movilidad costal alta y clavicular y estimulación de la musculatura abdominal $(9,10)$. En las revisiones sistemáticas realizadas por el grupo Cochrane (10), muestran que las técnicas respiratorias empleadas en el tratamiento del asma son: los ejercicios de reeducación del patrón respiratorio, el entrenamiento físico de los músculos respiratorios y periféricos, las técnicas espiratorias y ayudas instrumentales de limpieza mucociliar, el ejercicio aeróbico y las técnicas complementarias, lo que implica un gran trabajo de reeducación muscular por parte de paciente que muchas veces no tiene facilidad de entender o ejecutarlos de manera correcta, es ahí donde el VNM actuaría como herramienta complementaria por los efectos que genera su aplicación.

El objetivo de este estudio fue determinar el efecto del vendaje neuromuscular (VNM) como herramienta complementaria a la terapia respiratoria de adultos con asma y describir los parámetros respiratorios fisiológicos y espirométricos hallados en la población de estudio, dado que no hemos hallado estudios similares en nuestro país.

\section{MATERIAL Y METODOS}

El estudio realizado fue observacional, comparativo, longitudinal, y prospectivo. Se realizó en pacientes adultos con asma moderada, atendidos de manera ambulatoria en el Programa de Rehabilitación Cardiorrespiratoria del Hospital Cayetano Heredia durante el último trimestre del año 2015 y el primer trimestre del año 2016, que cumplieron con los criterios de inclusión: paciente con diagnóstico de asma, adulto entre 40 y 60 años, atendido en el Programa de Rehabilitación Cardiorrespiratorio del Hospital Cayetano Heredia durante el último trimestre del año 2015 y el primer trimestre del año 2016, que firmó el consentimiento informado para participar en el estudio. Se excluyeron pacientes con patologías respiratorias concomitantes como EPOC, alergias, neumonías, atelectasias y antecedente de tabaco.

Se incluyó un grupo de 20 pacientes en quienes se complementó la terapia respiratoria convencional con la aplicación de vendaje neuromuscular, material donado por los fisioterapeutas del servicio de rehabilitación respiratoria para ese número de pacientes; asimismo, se incluyó otro grupo de 20 pacientes que solo recibió la fisioterapia respiratoria convencional. 


\section{Tabla 1. Plan de tratamiento de la Terapia Respiratoria Convencional}
1. Técnicas de desobstrucción bronquial
2. Técnicas de reeducación ventilatoria.
3. Técnicas de aumento de la distensibilidad pulmonar
4. Ejercicios de débito inspiratorio controlado (EDIC)
5. Vibraciones manuales
6. Acondicionamiento físico
7. Técnicas de relajación y control respiratorio

La terapia respiratoria convencional (tabla 1) se aplicó en 10 sesiones de 45 minutos, dos veces por semana, lunes y viernes, mediante técnicas de higiene postural y reeducación ventilatoria, vibraciones manuales, desobstrucción bronquial, ejercicios de débito inspiratorio controlado (EDIC) y distensibilidad pulmonar, según el protocolo del servicio. Desde la sexta sesión se agregó como ayuda instrumental el uso de bandas elásticas para el acondicionamiento físico. Adicionalmente, a un grupo se le aplicó VNM (tabla 2) los días lunes al terminar la fisioterapia respiratoria convencional, con el cual retornaron a sus casas con la indicación de no retirar el vendaje, ni durante el aseo, aunque sin restricciones en sus actividades de la vida diaria; al cuarto día de cada aplicación (viernes), el fisioterapeuta les retiró el VNM, con el objetivo que la piel se regenere; el ciclo se repitió cada lunes hasta culminar las 10 sesiones programadas.

La aplicación del VNM se realizó con el paciente en posición decúbito supino (figura 1), con el área abdominal descubierta desde la apófisis xifoides hasta cuatro dedos por debajo del ombligo. La cinta del VNM se dobló en 2 partes iguales, utilizando la técnica de aplicación en "I"; luego se cortaron los bordes de la cinta en forma circular (sin puntas) y se pegó el centro de la cinta del VNM a la altura de la apófisis xifoides; con el paciente en inspiración máxima se estira cada extremo del VNM en dirección a los bordes de las costillas hasta llegar por debajo de la altura del ombligo. La tensión del VNM aplicada en la primera sesión fue del $10 \%$ de la longitud total del vendaje, incrementándose progresivamente en cada aplicación hasta un máximo del $40 \%$ (figura 2). La

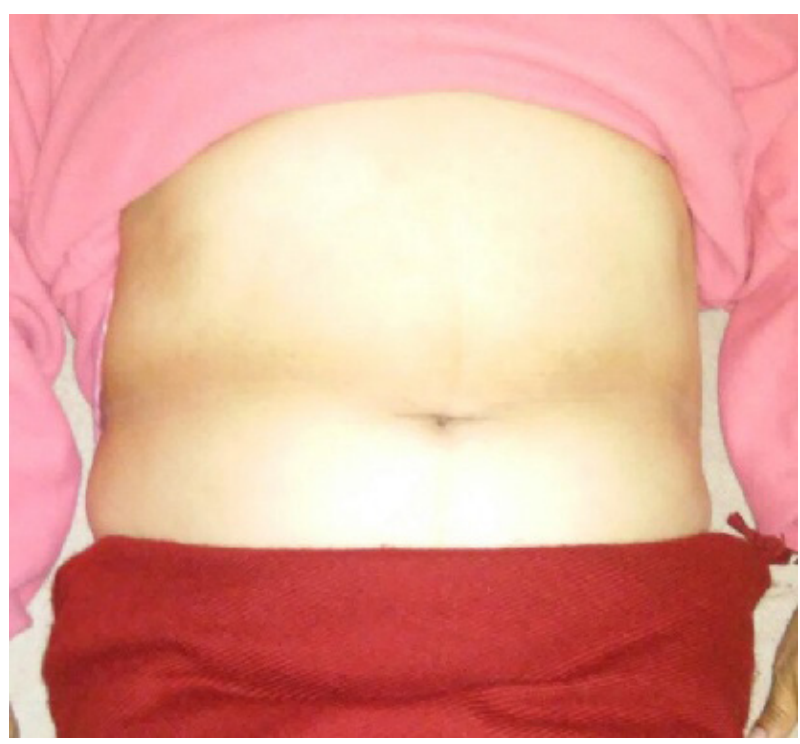

Figura 1. Localización del paciente en decúbito supino y con zona abdominal descubierta.

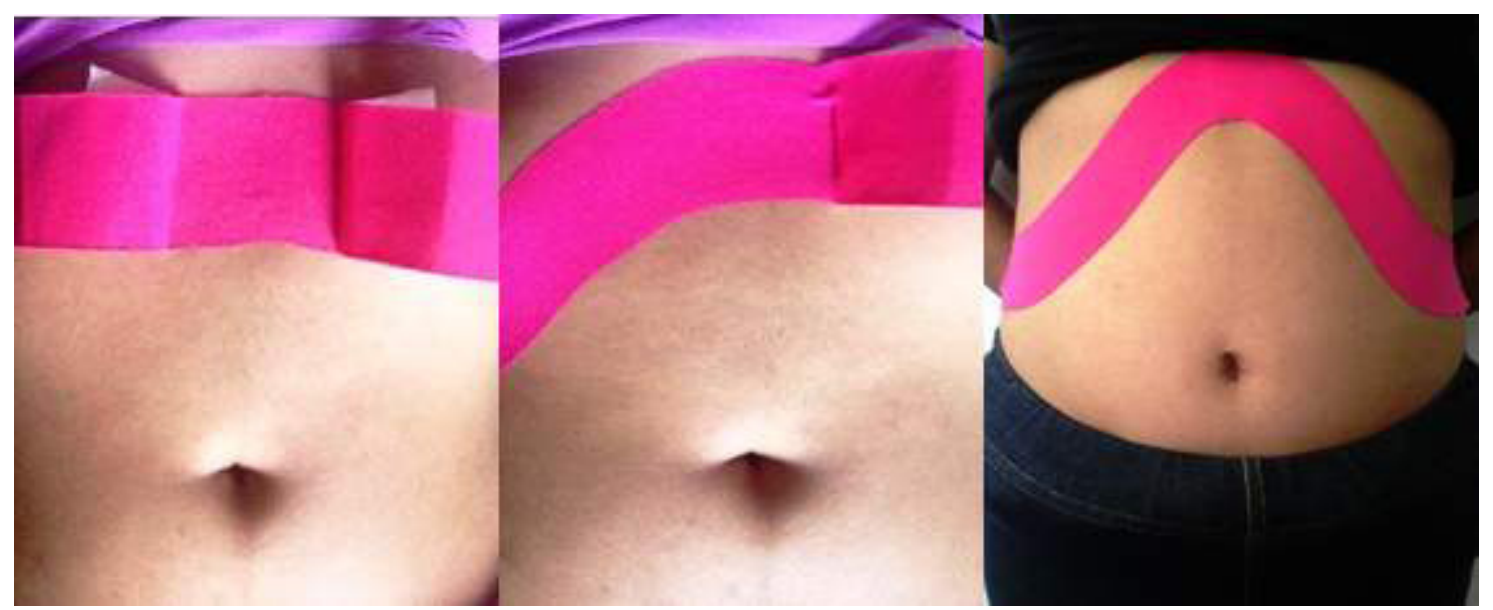

Figura 2. Procedimiento de aplicación del VNM en zona diafragmática. 
Tabla 2. Plan de tratamiento de la Terapia Respiratoria Convencional y VNM

\section{Terapia Respiratoria Convencional}

Técnicas de desobstrucción bronquial

Técnicas de reeducación ventilatoria.

Técnicas de aumento de la distensibilidad pulmonar

Ejercicios de débito inspiratorio controlado (EDIC)

Vibraciones manuales

Acondicionamiento físico

Técnicas de relajación y control respiratorio

\section{Aplicación del VNM en la zona del diafragma}

Acomodar al paciente en posición decúbito supino

Descubrir el área abdominal y limpiar con alcohol la zona de aplicación (diafragma)

La cinta del VNM se dobla en dos partes iguales, y se cortan los bordes de la cinta (en punta) en forma circular.

Se toma desde los extremos de la cinta (sin tocar el pegamento) y se pega la parte central de la cinta a la altura de la apófisis xifoides.

Se le pide al paciente una inspiración profunda, y se estira cada extremo de la cinta con el debido cuidado en dirección a los bordes de las costillas hasta llegar por debajo de la altura del ombligo.

aplicación fue realizada por un Tecnólogo Medico en Terapia Física y Rehabilitación con certificación en la técnica de aplicación del Vendaje Neuromuscular.

Para el presente estudio, recolectamos la información de interés (parámetros) en cada sesión, a partir de las fichas de seguimiento de terapia respiratoria, así como de las historias clínicas de los pacientes incluidos en el Programa Ambulatorio de Rehabilitación Cardiorrespiratoria. Todos los pacientes en el programa contaron con una evaluación respiratoria funcional y espirométrica al inicio y al final del tratamiento de rehabilitación, cuyos resultados se registraron en la ficha de atención, incluyendo $\mathrm{CV}$, VEF1, PEF, FC, FR, SO2 y patrón respiratorio; y se recolectaron de la historia clínica los datos edad, sexo, lugar donde reside el paciente y antecedente familiar de asma.

\section{RESULTADOS}

Se evaluaron 40 pacientes asmáticos, de quienes 20 recibieron VNM como tratamiento complementario al tratamiento convencional, y 20 que solo recibieron el tratamiento convencional, todos con seguimiento completo durante 10 sesiones de tratamiento de terapia respiratoria y con evaluación al inicio y final de la misma. Todos los pacientes incluidos, en ambos grupos, fueron de sexo femenino.

La edad de los pacientes estuvo entre los 42 y 59 años, con promedio de $46,8(\mathrm{DE}=2,5)$ en el grupo $\sin$
VNM y 46,4 $(\mathrm{DE}=4,0)$ en el grupo con VNM, sin diferencia estadísticamente significativa $(p=0,63)$. Asimismo, no hubo diferencia significativa en el antecedente familiar de asma ni la procedencia de los pacientes según uso de vendaje neuromuscular (tabla $3)$.

Antes del inicio de la terapia respiratoria, en los parámetros fisiológicos, el promedio de la $\mathrm{CV}$ fue mayor en el grupo que recibió $\mathrm{VNM}(\mathrm{p}=0,022)$, pero no hubo diferencia en el VEF ni el PEF (tabla 4). En los parámetros respiratorios fisiológicos, la frecuencia respiratoria fue menor en el grupo $\sin \operatorname{VNM}(p<0,05)$, pero sin diferencia en la frecuencia cardiaca ni saturación de oxígeno. El patrón respiratorio inicial fue torácico en todos los pacientes al inicio del tratamiento, en ambos grupos.

Si bien ambos grupos tuvieron mejoría al final del tratamiento, ésta fue mayor en el grupo que utilizó el VNM con una diferencia estadísticamente significativa $(p<0,01)$, tanto en los parámetros respiratorios espirométricos (tabla 5) donde la $\mathrm{CV}$, el VEF1 y el PEF mostraron mayor incremento, como en los parámetros fisiológicos respiratorios (tabla 6), donde hubo mayor disminución de la FC y FR, así como mayor incremento en la $\mathrm{SO}_{2}$.

En referencia al patrón respiratorio (PR), el 100\% de los pacientes de ambos grupos presentó patrón respiratorio torácico, pero en la evaluación post tratamiento hubo mayor mejoría en el grupo con 
Tabla 3. Antecedentes familiares de asma

\begin{tabular}{lccc}
\hline & Grupo sin VNM f (\%) & Grupo con VNM f (\%) & P* \\
\hline Antecedente & & & \\
Familiar de asma & $10(50 \%)$ & $9(45 \%)$ & 0.75 \\
Procedencia & & & 0.284 \\
Este & $7(70,0)$ & $3(30,0)$ & \\
Norte & $9(45,0)$ & $11(55,0)$ & \\
Sur & $4(50,0)$ & $4(50,0)$ & \\
Oeste & $0(0,0)$ & $2(100,0)$ & \\
\hline *Prueba Chi cuadrado & & &
\end{tabular}

Tabla 4. Valores de los parámetros respiratorios al iniciar el tratamiento

\begin{tabular}{lccc}
\hline $\begin{array}{c}\text { Parámetros antes del } \\
\text { tratamiento }\end{array}$ & $\begin{array}{c}\text { Grupo sin VNM } \\
\mathbf{x}(\mathbf{D E})\end{array}$ & $\begin{array}{c}\text { Grupo con VNM } \\
\mathbf{x}(\mathbf{D E})\end{array}$ & P* \\
\hline $\mathrm{CV}_{\mathrm{i}}$ & $3084,0 \mathrm{ml}(55,9)$ & $3119,9 \mathrm{ml}(53,8)$ & $<0.05$ \\
$\mathrm{VEF}_{\mathrm{i}}$ & $843,0 \mathrm{ml}(24,8)$ & $842,3 \mathrm{ml}(23,3)$ & 0.536 \\
$\mathrm{PEF}_{\mathrm{i}}$ & $2105,6 \mathrm{ml}(48,7)$ & $2119,8 \mathrm{ml}(60,3)$ & 0.208 \\
$\mathrm{FC}_{\mathrm{i}}$ & $84,4 \mathrm{lpm}(3,4)$ & $86,9 \mathrm{lpm}(4,3)$ & $<0.05$ \\
$\mathrm{FR}_{\mathrm{i}}$ & $30,7 \mathrm{rpm}(2,7)$ & $30,5 \mathrm{rpm}(3,2)$ & 0.583 \\
$\mathrm{SO}_{\mathrm{i}}$ & $93,5 \%(0,7)$ & $93,5 \%(0,7)$ & 0.590 \\
${ }^{*}$ Prueba t de student & & &
\end{tabular}

Tabla 5. Valores de los parámetros respiratorios al final del tratamiento

\begin{tabular}{lccc}
\hline $\begin{array}{c}\text { Parámetros después } \\
\text { del tratamiento }\end{array}$ & $\begin{array}{c}\text { Grupo sin VNM } \\
\mathbf{x}(\mathbf{D E})\end{array}$ & $\begin{array}{c}\text { Grupo con VNM } \\
\mathbf{x}(\mathbf{D E})\end{array}$ & P* \\
\hline $\mathrm{CV}_{\mathrm{f}}$ & $3299.1 \mathrm{ml}(55,7)$ & $3507,3 \mathrm{ml}(72,4)$ & $<0.01$ \\
$\mathrm{VEF}_{\mathrm{f}}$ & $900,0 \mathrm{ml}(13,7)$ & $962,3 \mathrm{ml}(19,7)$ & $<0.01$ \\
$\mathrm{PEF}_{\mathrm{f}}$ & $2387,5 \mathrm{ml}(128,6)$ & $2806.7 \mathrm{ml}(119,5)$ & $<0.01$ \\
$\mathrm{FC}_{\mathrm{f}}$ & $74,4 \mathrm{pm}(2,6)$ & $63,9 \mathrm{pm}(3,2)$ & $<0.01$ \\
$\mathrm{FR}_{\mathrm{f}}$ & $25 \mathrm{rpm}(0.8)$ & $21.1 \mathrm{rpm}(1.4)$ & $<0.01$ \\
$\mathrm{SO}_{\mathrm{f}}$ & $96,8 \%(0.7)$ & $98,2 \%(0.7)$ & $<0.01$ \\
\hline${ }^{*}$ Prueba t de student & & &
\end{tabular}

Tabla 6. Diferencia en parámetros respiratorios antes y después del tratamiento

\begin{tabular}{lccc}
\hline Parámetros antes-después (i-f) & Grupo sin VNM & Grupo con VNM & $\mathbf{P}^{*}$ \\
\hline $\mathrm{CV}_{\mathrm{i}-\mathrm{f}}$ & $215,2 \mathrm{ml}(+7.0)$ & $387,5 \mathrm{ml}(+12.4)$ & $<0.01$ \\
$\mathrm{VEF}_{\text {i-f }}$ & $57 \mathrm{ml} \mathrm{(+6.8)}$ & $120 \mathrm{ml}(+14.2)$ & $<0.01$ \\
$\mathrm{PEF}_{\mathrm{i}-\mathrm{f}}$ & $282 \mathrm{ml}(+13.4)$ & $686,9 \mathrm{ml}(+32.4)$ & $<0.01$ \\
$\mathrm{FC}_{\mathrm{i}-\mathrm{f}}$ & $-10,0 \mathrm{lpm}(-11.9)$ & $-23,0 \mathrm{lpm}(-26.5)$ & $<0.01$ \\
$\mathrm{FR}_{\mathrm{i}-\mathrm{f}}$ & $-5,7 \mathrm{rpm}(-18.4)$ & $-9,4 \mathrm{rpm}(-30.7)$ & $<0.01$ \\
$\mathrm{SO}_{\text {i-f }}$ & $3,25 \%(+3.5)$ & $4,70 \%(+5.0)$ & $<0.01$ \\
\hline${ }^{*}$ Prueba t de student & & &
\end{tabular}


VNM, donde los 20 pacientes (100\%) modificaron su patrón respiratorio a diafragmático, sin embargo, sólo 15 (75\%) de pacientes del grupo sin VNM mejoraron al patrón respiratorio de torácico a diafragmático (prueba exacta de Fisher $=7,059, \mathrm{p}=0,01$ ).

\section{DISCUSIÓN}

La aplicación del Vendaje Neuromuscular en la zona diafragmática, también llamado, "Kinesiotaping" o "Medical taping", facilita el incremento de la movilidad del diafragma(4,5), y ésta a su vez facilita el patrón respiratorio diafragmático en lugar de una respiración costal superior acentuada que, por la rigidez de la parrilla costal hace que los volúmenes respiratorios se encuentren disminuidos; así, al reducir la resistencia en la vía aérea y aumentar el drenaje mucociliar, se produciría un aumento en la capacidad vital. Esto corresponde con nuestros resultados, donde la aplicación del VNM estuvo asociada al incremento de la capacidad vital en $12,4 \%$ en el grupo que lo recibió en comparación con del grupo que no lo recibió en quienes solo aumentó en un 7\% al final del tratamiento. La mejoría evidenciada con la aplicación del VNM corresponde con lo encontrado por diversos autores como Szczegielniak y col., (11), que evidenciaron mejoría en CVF, VEF1, FEM25, FEM50, FEM75 y PEF, con el mayor efecto a las 24 horas de aplicarse VNM en una serie de 10 pacientes asmáticos; Meneses (12), encontró que el 40\% de los asmáticos en un programa de rehabilitación respiratoria aumentaron por encima del $15 \%$ el valor inicial de los volúmenes y las capacidades. Sin embargo, Boff R. (13) no encontró diferencia significativa en los valores de VEF1 y PEF; esto puede deberse a que el tiempo y la técnica de aplicación fueron distintas.

Estudios en sujetos sanos, como el realizado por Hombrados-Hernández et al., (14), concluyeron que la aplicación del VNM sobre el diafragma anterior no tiene efectos significativos, evaluados mediante la prueba de cicloergoespirometría y la prueba de 6MM; asimismo Zübeyir et al. (15), tampoco obtuvieron diferencias significativas en la fuerza muscular respiratoria después de la aplicación de VNM en el músculo diafragma y músculos respiratorios accesorios en estudiantes. Es posible que la diferencia de estos resultados con los nuestros se deba a la condición de enfermedad, donde un paciente puede tener un rango de mejora a diferencia de un paciente sano, en quienes el VNM y la terapia respiratoria podrían sí ejercer cambios fisiológicos y espirométricos en la mejora de la función pulmonar.
Boff (13) encontró que en el grupo de pacientes con EPOC que fueron tratados con VNM, tuvo una mejoría estadísticamente significativa en los niveles de SO2, lo que corresponde con nuestros resultados. Asimismo, Szczegielniak y col. (11), evidenciaron mejoría en la disnea, lo cual corresponde con la mayor mejora de patrón respiratoria evidenciada en el grupo que recibió VNM.

Si bien es cierto, en el estudio no se consideró la evaluación de la fuerza muscular del diafragma ni de los músculos accesorios de la respiración por razones de evitar subjetividad en los resultados, se consideraron los demás parámetros respiratorios (capacidad vital, VEF1, PEF, frecuencia cardiaca, frecuencia respiratoria, patrón respiratorio y saturación de oxígeno) por ser más objetivos en la medición, lo que permitió visualizar y comparar la mejoría real y significativa de los grupos.

\section{CONCLUSIONES}

En conclusión, se evidencia mayor mejoría tanto en los parámetros fisiológicos respiratorios como espirométricos con el uso de vendaje neuromuscular como terapia adicional al tratamiento convencional, donde el VNM estaría actuando como un facilitador en el trabajo diafragmático y costal favoreciendo el mínimo esfuerzo muscular, una mayor efectividad y un adecuado feedback neuromuscular, generando una mejor condición del paciente.

Es recomendable realizar nuevos estudios que evalúen la eficacia y seguridad del VNM como complemento a la fisioterapia respiratoria en pacientes asmáticos, según severidad y grupos etarios, así como evaluar su utilidad en otras patologías respiratorias. Se sugiere considerar la implementación del uso del VNM como complemento del tratamiento conservador de la terapia respiratoria en los centros de atención hospitalaria, previa capacitación de los fisioterapeutas.

\section{Correspondencia}

Ivette G. Ramos Bendezú

Correo electrónico: ivette.ramos@upch.pe

\section{REFERENCIAS BIBLIOGRAFICAS}

1. Aguirre T. Kinesiology Taping. Teoría y práctica. Andoain: Biocorp Europa; 2010.

2. Villota-Chicaíza XM. Vendaje neuromuscular: Efectos Neurofisiológicos y el papel de las fascias. Rev Ciencias de la Salud. 2014; 12: 253-269. 
3. Couto ER, Figueiredo FA, Augusto PC, Davi SF. Bandagem elástica associada à fisioterapia respiratória convencional en pacientes com doençia pulmonar obstrutiva crónica. Movim \& saúde. 2013; 26(6): 1822.

4. Jiménez I. Utilización del vendaje neuromuscular en el diafragma anterior en niños con discapacidad motriz grave y trastornos respiratorios asociados: A propósito de dos casos clínicos. Noticias de Vendaje Neuromuscular. 2011; 5:3-6.

5. Van Zuilen M. El uso de vendaje neuromuscular para incrementar el nivel de oxigenación en pacientes con neumopatía crónica. Noticias de Vendaje Neuromuscular. 2009; 2:4-7.

6. Global Asthma Network. Global Asthma Report 2014. New Zealand:Global Asthma Network; 2015.

7. Jardim JR, Mayer AF, Camelier A. Músculos respiratorios y rehabilitación pulmonar en asmáticos. Arch Bronconeumol. 2002; 38(4):181-8.

8. Global Initiative for Asthma. Pocket guide for asthma management and prevention (for adults and children older than 5 years). Global Initiative for Asthma; 2016.
9. Mezei G. Physiotherapy of asthma. Acta Microbiol Immunol Hung. 1998;45(1):157-66.

10. Holloway E, Ram FS. Breathing exercises for asthma. Cochrane Database Syst Rev 2004;1. DOI: 10.1002/14651858.CD001277.pub2

11. Szczegielniak J, £uniewski1 J, Bunio A, Bogacz K, Eliwiñski Z. Zastosowanie kinesio tapingu $\mathrm{u}$ pacjentów z zaostrzeniami astmy oskrzelowej. Med Sport. 2007;23(6):337-41.

12. Meneses MR. Programa de rehabilitación respiratoria en pacientes asmáticos: repercusión sobre las pruebas funcionales respiratorias. Rev Cuba Med. 1999;38(3):178-182.

13. Daitx, R Kinesio taping como terapia complementar no tratamento fisioterapêutico de pacientes com exacerbação de doença pulmonar obstrutiva crónica. Tesis de Maestria. Santa Catarina:UNISUL; 2015.

14. Hombrados-Hernández, E. Segura-Ortí, M.A. BuilBellver. Efectos de la aplicación del Kinesio taping ${ }^{\mathrm{TM}}$ en el diafragma en el resultado la cicloergoespirometría y la prueba marcha de 6 minutos. Fisioterapia.2011; 33(2):64-69.

15.Zübeyir S, Nilüfer K, Burcu C, et al.: The effect of kinesiology taping on respiratory muscle strength. J Phys Ther Sci. 2009; 24: 241-244. 